\title{
The Path of a Pseudo-Triangulation
}

\author{
Oswin Aichholzer* $\quad$ Bettina Speckmann ${ }^{\dagger} \quad$ Ileana Streinu ${ }^{\ddagger}$
}

\begin{abstract}
We define the path of a pseudo-triangulation, a data structure generalizing the path of a triangulation of a point set. This structure allows us to use divide-andconquer type of approaches for suitable (i.e. decomposable) problems on pseudo-triangulations. We illustrate this method by presenting a novel algorithm that counts the number of pseudo-triangulations of a point set.
\end{abstract}

\section{Introduction}

Pseudo-triangulations, unlike triangulations, only recently emerged as a promising data structure with a variety of applications. They were originally introduced in the context of visibility complexes and ray shooting. But in last few years they also found application in robot arm motion planning and kinetic collision detection. In particular the so-called minimum or pointed pseudo-triangulations introduced by Streinu [2] exhibit many fascinating properties that initiated a growing interest in their geometric and combinatorial nature.

The path of a triangulation was introduced by Aichholzer [1] who used it to efficiently count all triangulations of a planar point set in a divide-and-conquer like manner. Since pseudo-triangulations and triangulations share certain properties, it is an obvious questions to ask if there is a meaningful extension of the path concept to pseudo-triangulations.

In this abstract we propose a definition of a path of a pseudo-triangulation, which retains many of the useful properties of a triangulation path. Specifically, we show that pseudo-triangulation paths like triangulation paths can be used in an efficient divide-and-conquer like approach to count the number of pseudo-triangulations of a point set.

\footnotetext{
* Institute for Software Technology, Graz University of Technology, Inffeldgasse 16b, A-8010 Graz, Austria, oaich@ist.tu-graz.ac.at. Research partially supported by APART [Austrian Programme for Advanced Research and Technology] of the Austrian Academy of Sciences.

${ }^{\dagger}$ Institute for Theoretical Computer Science, ETH Zürich, CH8092 Zürich, Switzerland, speckman@inf.ethz.ch. Research supported by the Berlin-Zürich Graduate Program "Combinatorics, Geometry, and Computation", financed by ETH Zürich and the German Science Foundation (DFG).

${ }^{\ddagger}$ Department of Computer Science, Smith College, Northampton, MA 01063, USA, streinu@cs.smith.edu. Research supported by NSF grant CCR-0105507.
}

\section{The Path of a Simple Polygon}

Let us assume that $P$ is a simple polygon that may also contain internal points. We denote the boundary of $P$ by $B(P)$ and the set of vertices of $P$ (including its internal points) by $V(P)$. We assume that all points in $V(P)$ are in general position.

A pseudo-triangle is a planar polygon that has exactly three convex vertices, called corners, with internal angles less than $\pi$. Three concave chains, called sides, join the three corners. A pseudo-triangulation $\mathcal{T}(P)$ of $P$ is a partition of the interior of $P$ into pseudo-triangles whose vertex set is exactly $V(P)$.

Observation 1 Let $P$ be a simple polygon with $c$ convex vertices on $B(P)$ and $i$ internal points. A pseudotriangulation of $P$ is pointed iff it contains $c-2+i$ pseudo-triangles.

Any simple polygon has at least one pointed pseudo-triangulation. Assume that we are given such a pointed pseudo-triangulation $\mathcal{T}$ of $P$ and a line $l$ that intersects $P$. Let $E=\left\{e_{1}, \cdots, e_{k}\right\}$ be the set of edges of $\mathcal{T}$ that is crossed by $l$, ordered from top to bottom according to their intersection with $l$ (w.l.o.g. we assume $l$ not to be horizontal). Consider a pair of consecutive edges $e_{i}$ and $e_{i+1}$ in $E$. We denote by the lean of the pair $\left(e_{i}, e_{i+1}\right)$ the location of the intersection of their supporting lines with respect to $l$, i.e., if the supporting lines of $e_{i}$ and $e_{i+1}$ intersect to the left (resp. right) of $l$ then we say that $\left(e_{i}, e_{i+1}\right)$ leans to the left (resp. right). An edge $e_{i}$ for which the lean of $\left(e_{i-1}, e_{i}\right)$ and $\left(e_{i}, e_{i+1}\right)$ differs is called a signpost (see Fig. 1 (a)-(b)).

Let $T$ be the set of the pseudo-triangles crossed by $l$. We remove all edges of $E$ that are not signposts and merge their adjacent pseudo-triangles. Let $T^{*}$ denote the resulting set of polygons. The line $l$ crosses the polygons in $T^{*}$ exactly at the signposts (see Fig. 1 (c)).

We now construct the pseudo-triangulation path for $\mathcal{T}$ with respect to $l, \pi_{l}(\mathcal{T})$, by joining adjacent signposts along the boundary of their common face in $T^{*}$ according to their lean, i.e., if two adjacent signposts lean to the left, then we connect them via the edges of their common polygon that lie to the left of $l$ (see Fig. 1 (d)). We denote by $e_{s} \in B(P)$ the start edge and correspondingly by $e_{e} \in B(P)$ the end edge of a pseudo-triangulation path.

We say that a pseudo-triangulation path is non-trivial if it contains at least one edge that is not part of $B(P)$. 


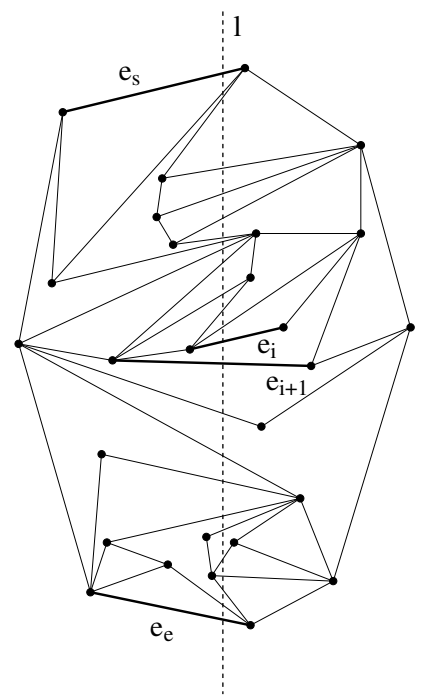

(a) A pseudo-triangulation cut by a line $l$ - the pair $\left(e_{i}, e_{i+1}\right)$ leans to the left.

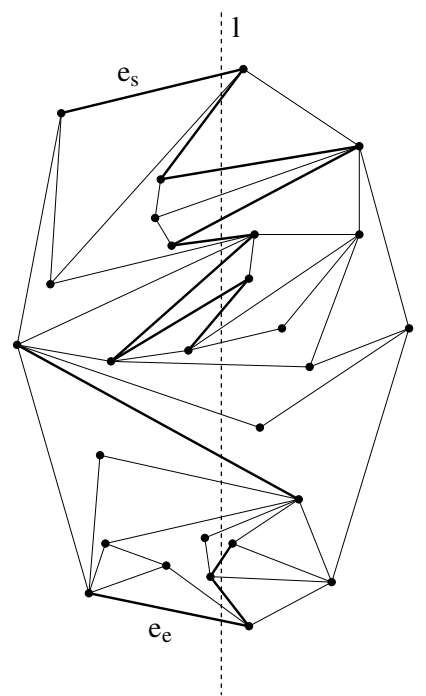

(b) The signposts.

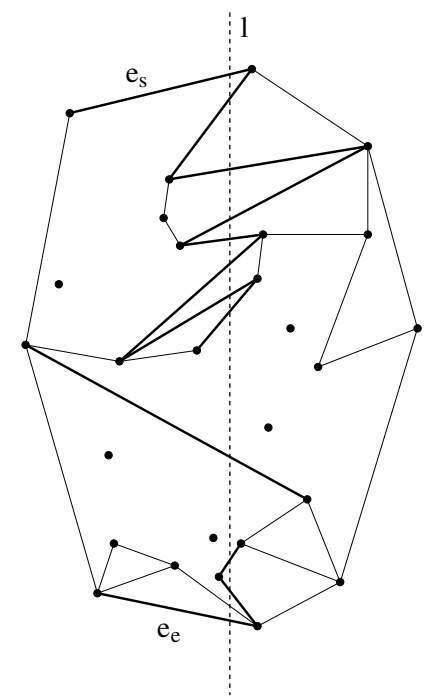

(c) Removing edges that are cut by $l$ but are not signposts.

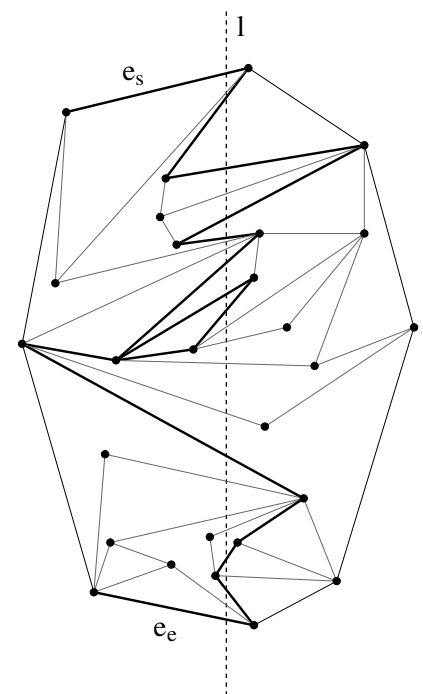

(d) The path of this pseudo-triangulation.

Figure 1: Constructing the path of a pseudo-triangulation.

Furthermore, we denote by $\pi_{l}(P)$ the set of all paths for a simple polygon $P$ with respect to a line $l$.

\section{Properties}

Property 1 Consecutive pairs of signposts alternate in their lean.

\section{Property 2 (Empty Area Property)}

The area bounded by the path between (and including) two consecutive signposts and the line $l$ is an empty pseudo-triangle.

\section{Property 3 (Non-empty Wedge Property)}

The supporting lines of two consecutive signposts define four wedges, three of which are crossed by $l$. The closed region of the forth wedge contains at least one point $p$ of $P$.

Property 4 Each pseudo-triangulation path in $\pi_{l}(P)$ can be completed to a pointed pseudo-triangulation of $P$.

Lemma 1 Two pseudo-triangulation paths are either identical, or properly intersect each other, or one violates the pointedness property at at least one vertex of the other.

Theorem 2 (Uniqueness) The path $\pi_{l}(\mathcal{T})$ for a pointed pseudo-triangulation $\mathcal{T}$ of a simple polygon $P$ with respect to a line $l$ is well-defined and unique.

Lemma 3 Assume we are given a partial path $\pi^{*}$ in $P$, i.e., a path that starts at the start-edge and ends at an signpost, then there always exists a path $\pi$ in $\pi_{l}(P)$ such that $\pi^{*}$ is a sub-path of $\pi$.
For a set $S$ of points in the plane a line $l$ is feasible if no line spanned by two points of $S$ is parallel to $l$.

Lemma 4 If $B(P)$ contains at least 4 convex vertices or if $P$ contains at least one inner point, then for each given feasible direction there exists a line $l$ such that all path in $\pi_{l}(P)$ are non-trivial.

\section{Generating pseudo-triangulation paths}

In this section we (very) briefly sketch how to generate all pseudo-triangulation paths for a given polygon $P$. We can then apply the generator recursively to count all pointed pseudo-triangulations of a given set of points.

Let $e_{s}, e_{e} \in B(P)$ be the start- and ending edge, respectively, of any path in $\pi_{l}(P)$. Starting with $e_{s}$ we iteratively continue a (partial) path $\pi^{*} \in \pi_{l}(P)$ by adding a new signpost and properly connecting it to the previous signpost until we reach $e_{e}$. By considering all possible choices of signposts and all ways of pairwise connecting them we will thus generate the entire set $\pi_{l}(P)$.

Theorem 5 For a polygon $P$ and line $l$ the set $\pi_{l}(P)$ of all pseudo-triangulation paths can be generated in $O\left(n^{4} \log ^{2} n\left|\pi_{l}(P)\right|\right)$ time.

\section{References}

[1] O. Aichholzer. The Path of a Triangulation. In Proc. 15th ACM Symp. Computational Geometry, pages 14-23, 1999.

[2] I. Streinu. A combinatorial approach to planar non-colliding robot arm motion planning. In Proc. 41st FOCS, pages 443$453,2000$. 